\title{
Linear growth harmonic functions on complete manifolds
}

\author{
JIAPING WANG
}

\section{Introduction.}

Let $(M, g)$ be a complete noncompact connected Riemannian manifold with the associated Laplace-Beltrami operator $\triangle$. Then harmonic functions of $M$ are the solutions to the equation $\Delta f=0$. The first significant theorem about harmonic functions on complete manifolds was proved by Yau $[\mathrm{Y}]$ around 1974. He showed that any positive harmonic function defined on $M$ must be identically constant if the Ricci curvature of $M$ is nonnegative. In other words, the strong Liouville property is true on such a manifold. His argument relied on estimating the gradient of positive harmonic functions, of which a local version was developed later in his joint work with Cheng in [C-Y] (see [L-Y] for the parabolic version). Using this local version of gradient estimate, one can actually show that there does not exist any nonconstant sublinear growth harmonic function on a complete manifold with nonnegative Ricci curvature as observed by Cheng. Namely, if $M$ is a complete Riemannian manifold with nonnegative Ricci curvature and $f$ is a harmonic function on $M$ which satisfies

$$
|f|(y) \leq o(r(y))
$$

for some distant function $r$ to a fixed point, then $f$ must be identically constant. Note that the polynomial growth harmonic functions on $R^{n}$ are spanned by the set of harmonic polynomials of integral degree. In particular, as far as sublinear growth harmonic functions are concerned, manifolds with nonnegative Ricci curvature are exactly the same as $R^{n}$. This led Yau to formulate the following conjecture.

Conjecture (Yau). For each integer $p$, the space $H^{p}(M)$ of harmonic functions on manifold $M$ with nonnegative Ricci curvature satisfying

$$
|f|(y) \leq O\left(r^{p}(y)\right)
$$


is finite dimensional.

The purpose of this paper is to study linear growth harmonic functions on a complete manifold whose Ricci curvature is allowed to be negative somewhere. Our motivation comes from the following naive approach to Yau's conjecture. Suppose that for each $p$ we can introduce a new Riemannian metric $h$ on $M$ such that the space $H^{p}(M, g)$ becomes part of $H^{1}(M, h)$, then to prove Yau's conjecture, it suffices to show that the space $H^{1}(M, h)$ is of finite dimension. However, since in general $(M, h)$ has nonconstant sublinear growth harmonic functions, its Ricci curvature must be negative somewhere in view of Cheng's result. Thus, it becomes necessary to consider the linear growth harmonic functions on a complete manifold with some negative curvature in order to take this approach. Here, we show that the space of linear growth harmonic functions on a manifold with nonnegative Ricci curvature outside a compact set has finite dimension provided that the first Betti number of the manifold is finite. Moreover, the dimension can be estimated in terms of the lower bound of the Ricci curvature and the diameter of the compact set. We hope that our result serves as an evidence to support Yau's conjecture.

Theorem 1.1. Let $M^{n}$ be an n-dimensional complete Riemannian manifold with nonnegative Ricci curvature outside ball $B_{p}(a)$, where $p \in M$ is a fixed point. Assume that the first Betti number of $M$ is finite. Then the space of all linear growth harmonic functions on $M$ has finite dimension. Moreover, if $\operatorname{Ric}_{M} \geq-K$ on $B_{p}(a)$ for some nonnegative number $K$, then there exists a constant $C(K, a, n)$ depending only on $K, a$ and $n$ such that the dimension is less than or equal to $C(K, a, n)$.

Unlike the case that the Ricci curvature is nonnegative everywhere, in general there exist nonconstant sublinear growth harmonic functions on a manifold with some negative curvature as surface examples show. A priori, it is unclear whether that the dimension of the space $H^{p}(M)$ as a function of $p$ can have only finite many jumps on the interval $[0,1]$. Our result indicates that this is the case.

When $M$ is a complete surface, the approach described above essentially works as shown independently by $\mathrm{Li}$ and Tam [L-T5] and Kasue [K3]. In particular, Yau's conjecture is true for complete surfaces. In fact, a more general class of surfaces, the surfaces with finite total curvature, was considered. Such surfaces were first studied by Huber $[\mathrm{H}]$. While Kasue gave an upper bound of $\operatorname{dim} H^{p}(M), \mathrm{Li}$ and Tam estimated $\operatorname{dim} H^{p}(M)$ both from below and above. Kasue also announced that Yau's conjecture is valid on a 
special class of manifolds. This class of manifolds is asymptotically locally Euclidean in the sense of $[\mathrm{B}]$. We refer to [L1] for the precise statement.

It is well-known that the first Betti number of an n-dimensional complete manifold $M$ with nonnegative Ricci curvature is at most $\mathrm{n}$. Therefore, $\operatorname{dim} H^{1}(M) \leq C(n)$ for some constant $C(n)$ which only depends on $n$. That was first proved by $\mathrm{Li}$ and Tam in [L-T4]. In fact, they gave a sharp estimate on the dimension of the space of all linear growth harmonic functions in terms of the order of volume growth. Namely, if the volume of the geodesic balls of radius $r$ centered at some point $x \in M$ satisfies $V\left(B_{x}(r)\right) \leq O\left(r^{k}\right)$, then $\operatorname{dim} H^{1}(M) \leq k+1$. In particular, the Bishop comparison theorem implies that $V\left(B_{x}(r)\right) \leq O\left(r^{n}\right)$, hence $\operatorname{dim} H^{1}(M)$ is at most $n+1$.

A special class of manifolds with nonnegative Ricci curvature outside a compact set is the connected sums of finitely many complete manifolds, each with nonnegative Ricci curvature. On such manifolds, Tam [T] has studied the linear growth harmonic functions and proved that the space of linear growth harmonic functions must be finite dimensional. In fact, he showed that if $M$ is the connected sum of $M_{i}, i=1, \ldots, k$, and each $M_{i}$ has nonnegative Ricci curvature, then $\operatorname{dim} H^{1}(M)=\sum_{i=1}^{k} \operatorname{dim} H^{1}\left(M_{i}\right)<$ $\infty$. Since the first Betti number of the connected sum of finitely many complete manifolds, each with nonnegative Ricci curvature, is also finite, we can recover part of Tam's result. On the other hand, if we assume $M$ has nonnegative sectional curvature outside a compact set, then $M$ is of finite topological type (see [L-T6] or [A]). Consequently, the first Betti number of $M$ must be finite. However, it is still unclear whether the first Betti number of a complete manifold with nonnegative Ricci curvature outside a compact set is finite or not in general.

Corollary 1.2. Let $M^{n}$ be an n-dimensional complete Riemannian manifold with nonnegative sectional curvature outside a compact subset $S$. Assume that the sectional curvature $K_{M} \geq-K$ on $S$ for some nonnegative number $K$, then there exists a constant $C(K, d, n)$ depending on $K, d$ and $n$ only such that the dimension of the space of all linear growth harmonic functions on $M$ is less than or equal to $C(K, d, n)$, where $d$ is the diameter of $S$.

The study of harmonic functions on manifolds with some negative curvature has been initiated by $\mathrm{Li}$ and Tam in [L-T6]. In that paper, they studied the bounded and positive harmonic functions on a manifold with nonnegative sectional curvature outside a compact subset. By first showing that such a manifold has finite topological type and thus finitely many ends, they 
defined the notions of large and small ends in terms of the volume growth of the end. With these definitions, their theorem asserts that both the bounded harmonic function space $H^{b}(M)$ and the space $H^{+}(M)$ spanned by positive harmonic functions are of one dimension if there is no large end. However, if there is at least one large end, then $\operatorname{dim} H^{b}(M)$ is equal to the number of large ends and $\operatorname{dim} H^{+}(M)$ equal to the number of ends which is finite. In particular, this implies that the Liouville property fails in general for a Riemannian manifold if its sectional curvature is negative somewhere. By constructing a metric at infinity (see [K1]) for asymptotically nonnegatively curved manifolds, Kasue later [K2] generalized Li and Tam's result to that situation. $M$ is asymptotically nonnegatively curved means that the sectional curvature $K$ of $M$ satisfies $K \geq-k(r(x))$ for some nonnegative monotone nonincreasing continuous function of the distance $r$ to a fixed point with the property that $\int_{1}^{\infty} t k(t) d t<\infty$. Recently, Li and Tam introduced the volume comparison (VC) property on general manifolds and studied positive Green's functions on a manifold $M$ with (VC) and whose Ricci curvature satisfies $\operatorname{Ric}_{M}(x) \geq-\frac{C}{1+r^{2}(x)}$, where $C>0$ is a constant and $r(x)$ is the distance function to a fixed point. As corollaries, they showed that part of the above results is also true on such a manifold. In the special case when $M$ has nonnegative Ricci curvature outside a compact set, then they proved that all conclusions mentioned above hold on $M$ if the first Betti number of $M$ is finite. We refer to their paper [L-T2] for more results and details.

When $M$ is only assumed to have nonnegative Ricci curvature outside a compact set, Donnelly $[D]$ observed that the dimension of the space of all bounded harmonic functions on such a manifold is necessarily finite. Cheng [C] later proved that the space spanned by all positive harmonic functions on such a manifold is also finite, and estimated the dimension of the space of bounded harmonic functions on $M$ in terms of the diameter and the lower bound of the Ricci curvature of the compact set. Both results were generalized by Li and Tam in [L-T1]. Among other things, they proved that $\operatorname{dim} H^{0}(M) \leq C(n, K)$ for some constant $C$ depending only on $n$ and $K$ if the Ricci curvature of $M$ is nonnegative on $M \backslash B_{p}(1)$ and bounded from below by $-K$ on $B_{p}(1)$ for some $K \geq 0$. Here, the space $H^{0}(M)$ is the linear space spanned by the set of harmonic functions on $M$ which are bounded on one side at each end. Combining this result with the fact that the number of ends of any manifold $N$ (without any curvature assumption) is always less than or equal to the dimension of the space $H^{0}(N)$ (see [L-T1]), they were able to conclude that if $M$ has nonnegative Ricci curvature outside a 
compact set, then $M$ has finite many ends. This was also proved by Cai [Cai] using a totally different approach.

The author would like to thank P. Li and L. F. Tam for their interest in this work and many helpful suggestions.

\section{Preliminaries.}

First we would like to recall some definitions and known facts which will be needed later on. Throughout, we let $M$ be a complete connected noncompact Riemannian manifold.

Definition 2.1. An end $E$ of a manifold $M$ is an unbounded component of the complement of some compact, smooth domain $D$ of $M$. In this case, we say that $E$ is an end corresponding to $D$.

For each such $D, M \backslash D$ has only finitely many components since $M$ is a connected manifold. The boundary of each component is smooth. In particular, each end has smooth boundary. It is also clear that if $D_{1} \subset D_{2}$ are compact, smooth domains of $M$, then the number of ends corresponding to $D_{1}$ is less than or equal to the number of ends corresponding to $D_{2}$. Hence, we say that $M$ has finitely many ends if there exists $b<\infty$ such that the number of ends corresponding to $D$ is less than or equal to $b$ for any compact, smooth domain $D \subset M$. One also observes that in this case there exist an integer $b_{0}<\infty$ and a compact, smooth domain $D_{0} \subset M$ such that the number of ends corresponding to $D$ is $b_{0}$ for all compact, smooth domain $D$ containing $D_{0}$. We say that $b_{0}$ is the number of ends of $M$. If there is no such $b_{0}$, then we say $M$ has infinite many ends.

The following result was proved in [L-T1].

Theorem 2.2 (Li, Tam). Let $M^{n}$ be a complete noncompact manifold and $p \in M$ be a fixed point such that $\operatorname{Ric}_{M}(x) \geq-k(r(x))$ where $r(x)=$ $r(p, x)$. Suppose that $k:[0, \infty) \rightarrow[0, \infty)$ is a nonincreasing continuous function such that $\int_{0}^{\infty} r^{n-1} k(r) d r<\infty$. Then the number of ends of $M$ is less than or equal to $C(n, k)$.

Definition 2.3. A Riemannian manifold $M$ with boundary $\partial M(\partial M$ may be empty) is said to be parabolic if it does not admit any positive Green's function satisfying Neumann boundary condition on $\partial M$. Otherwise, it is said to be non-parabolic. In particular, an end $E$ of $M$ is parabolic if it does not admit any positive Green's function satisfying Neumann boundary condition on $\partial E$, and nonparabolic otherwise. 
It has been shown in [L-T1] that an end $E$ is nonparabolic if and only if there exists a harmonic function $\phi$ defined on $E$ such that $0 \leq \phi \leq 1, \phi=1$ on $\partial E$ and $\inf _{E} \phi=0$. Moreover, the following result was proved recently by $\mathrm{Li}$ and Tam in [L-T2].

Lemma 2.4. Let $M$ be a complete noncompact manifold such that its Ricci curvature satisfies $\operatorname{Ric}(x) \geq-\frac{C}{(1+r(x))^{2}}$ for some nonnegative constant $C$. Suppose $M$ also satisfies condition (VC) defined in [L-T2]. Then on each nonparabolic end of $M$, there exists a unique harmonic function $\phi$ such that $0 \leq \phi \leq 1, \phi=1$ on $\partial E$ and $\lim _{x \rightarrow \infty} \phi=0$.

We also need the following fact (see [L-T2]).

Lemma 2.5. Let $M$ be a complete manifold with $k$ ends. Suppose that the first Betti number of $M$ is finite. For $R$ sufficiently large, and for $r>0$, let $B_{p}(R, R+r)$ denote the annulus $B_{p}(R+r) \backslash \overline{B_{p}(R)}$, and $M(R)$ be the union of all the unbounded components of $M \backslash \overline{B_{p}(R)}$. Then $B_{p}(R, R+r) \cap M(R)$ has exactly $k$ connected components.

The following lemma, which can be viewed as a generalization of a result in [L2], plays a crucial role in the proof of our main theorem.

Lemma 2.6. For $E$ a nonparabolic end of $M$ corresponding to the geodesic ball $B_{p}(a)$, let $E(R)$ be the unbounded component of $E \backslash \overline{B_{p}(R)}$ and $E(R, R+$ $r)=E(R) \cap B_{p}(R, R+r)$, where $B_{p}(R, R+r)$ denotes the annulus $B_{p}(R+r) \backslash$ $\overline{B_{p}(R)}$. Suppose that the Ricci curvature of $M$ satisfies Ric $(x) \geq-\frac{C}{(1+r(x))^{2}}$ for some nonnegative constant $C$ for all $x \in E$. Suppose also that the double of $E$ has finite first Betti number, and satisfies the condition (VC) in the sense of [L-T2]. Then for any bounded subharmonic function $f$ on $E$, we have

$$
\lim _{R \rightarrow \infty} \frac{1}{V_{E}(R, 2 R)} \int_{E(R, 2 R)} f=\limsup _{x \rightarrow \infty, x \in E} f
$$

where $V_{E}(R, 2 R)$ is the volume of the set $E(R, 2 R)$.

Proof. Since $E$ is nonparabolic, applying lemma 2.4 to the double of $E$, one concludes that there exists a harmonic function $g$ defined on $E$ such that $g=1$ on $\partial E$ and $\lim _{x \rightarrow \infty, x \in E} g(x)=0$. Using $g$ as a barrier function, one easily constructs a harmonic function $p$ on $E$ such that $p=f$ on $\partial E$ and $\lim _{x \rightarrow \infty, x \in E} p(x)=0$. Now define a function $w$ on $E$ by $w=p+c(\infty)(1-g)$, where $c(\infty)=\lim \sup _{x \rightarrow \infty, x \in E} f$. Then, $w=f$ on $\partial E$ 
and $\lim _{x \rightarrow \infty, x \in E} w(x)=\lim \sup _{x \rightarrow \infty, x \in E} f$. Consider $h=w-f$. Clearly, $h$ is superharmonic on $E, h=0$ on $\partial E$ and $\liminf _{x \rightarrow \infty, x \in E} h(x)=0$. If $h$ is constant on $E$, then $f$ converges to $c(\infty)$ at the infinity of $E$ and the lemma is trivially true. Thus, we may assume that $h$ is not a constant function on $E$. In view of the assumption on $E$ and lemma 2.5, there is a constant $R_{0}>a$ such that for $R \geq R_{0}, E(R, R+r)$ is connected for any $r>0$. If we let $c_{0}=\inf _{\partial E\left(R_{0}\right)} h$, then $c_{0}>0$ by the strong minimum principle for superharmonic functions. Since $\liminf _{x \rightarrow \infty, x \in E} h(x)=0$, $\liminf \operatorname{in}_{x \rightarrow \infty, x \in E\left(R_{0}\right)} h(x)=0$. Thus, for any $c_{0}>\epsilon>0$, there exists $R(\epsilon)>R_{0}$ such that $\inf _{E\left(R_{0}\right) \cap \partial B_{p}(R(\epsilon))} h(x) \leq \epsilon$. We claim that for any $R>R(\epsilon)$,

$$
\inf _{\partial E(R)} h(x) \leq \epsilon .
$$

In fact, on the set $S=E\left(R_{0}\right) \backslash E(R), \inf _{S} h(x) \leq \epsilon$ as $E\left(R_{0}\right) \cap \partial B_{p}(R(\epsilon)) \subset$ $S$. On the other hand, $S=E\left(R_{0}, R\right) \cup \cup_{i=1}^{m} t_{i}$, where $t_{i}, i=1, \ldots, m$, are the bounded components of $E\left(R_{0}\right) \backslash B_{p}(R)$. By the choice of $R_{0}, E\left(R_{0}, R\right)$ is connected. Also, each $t_{i}$ is connected and has nonempty intersection with $E\left(R_{0}, R\right)$. Thus, $S$ is a connected set and $\partial S=\partial E\left(R_{0}\right) \cup \partial E(R)$. However, $c_{0}=\inf _{\partial E\left(R_{0}\right)} h>\epsilon$. Therefore, by the minimum principle

$$
\inf _{\partial E(R)} h(x)=\inf _{\partial S} h(x)=\inf _{S} h(x) \leq \epsilon
$$

as $h$ is superharmonic.

Define $u(x, t)=\int_{E} H(x, y, t) h(y) d y$, where $H(x, y, t)$ is the heat kernel of $E$ satisfying Dirichlet boundary condition on $\partial E$. Arguing as in [L2] and noting that both $H$ and $h$ vanish on $\partial E$, we then conclude that

$$
\frac{\partial}{\partial t} u(x, t)=\int_{E} H(x, y, t) \triangle h(y) d y \leq 0 .
$$

Therefore, $u(x, t)$ is a monotone decreasing function of $t$. It is easy to see that the Ricci curvature of the double of $E$ satisfies $\operatorname{Ric}(x) \geq-\frac{c}{(1+r(x))^{2}}$ for some nonnegative constant $c$. Together with the fact that the double of $E$ satisfies condition (VC) we assert (see Lemma 1.4 in [L-T2]) that the set $E(R, 2 R)$ can be covered by $N$ number of balls $B_{y_{i}}\left(\frac{2 R}{5}\right)$ with centers $y_{i} \in E(R, 2 R)$, where $N$ is a constant independent of $R$. For $R$ sufficiently large, the set $E(R, 2 R)$ is connected and $\operatorname{Ric}(x) \geq-\frac{C}{R^{2}}$ on $E(R, 2 R)$. Thus, applying the Harnack inequality in [L-Y] to $u(x, t)$ on each ball $B_{y_{i}}\left(\frac{2 R}{5}\right)$ and by the connectedness of $E(R, 2 R)$, we conclude that $u\left(x, R^{2}\right) \leq c u\left(y, 2 R^{2}\right)$ for any $x, y \in E(R, 2 R)$, where $c$ is a constant independent of $R$. Since $\inf _{\partial E(R)} h(x) \leq \epsilon$ and $u(x, t)$ is a decreasing function of $t, u\left(x, R^{2}\right) \leq c \epsilon$ 
on $E(R, 2 R)$. On the other hand, by [SC], $H\left(y_{i}, x, R^{2}\right) \geq \frac{c}{V_{y_{i}}\left(\frac{2 R}{5}\right)}$ for any $x \in B_{y_{i}}\left(\frac{2 R}{5}\right)$, where $c$ is a constant independent of $R$. Therefore, for each $i=1, \ldots, N$,

$$
u\left(y_{i}, R^{2}\right) \geq \int_{B_{y_{i}}\left(\frac{2 R}{5}\right)} H\left(y_{i}, x, R^{2}\right) h(x) d x \geq \frac{c}{V_{y_{i}}\left(\frac{2 R}{5}\right)} \int_{B_{y_{i}}\left(\frac{2 R}{5}\right)} h(x) d x .
$$

Adding those $N$ inequalities together, we obtain

$$
N c \epsilon \geq \sum_{i=1}^{N} u\left(y_{i}, R^{2}\right) \geq \frac{c}{\sum_{i=1}^{N} V_{y_{i}}\left(\frac{2 R}{5}\right)} \int_{\cup_{i=1}^{N} B_{y_{i}}\left(\frac{2 R}{5}\right)} h(x) d x .
$$

Now pick up a point $x_{0} \in \partial E\left(\frac{3 R}{2}\right) \subset E(R, 2 R)$, then clearly $B_{x_{0}}\left(\frac{2 R}{5}\right) \subset$ $E(R, 2 R)$. By the Bishop volume comparison theorem and the connectedness of the set $E(R, 2 R)$, there is a constant $c$ independent of $R$ such that (see $[\mathrm{L}-\mathrm{T} 2])$

$$
V_{y_{i}}\left(\frac{2 R}{5}\right) \leq c V_{x_{0}}\left(\frac{2 R}{5}\right)
$$

for each $i=1, \ldots, N$. In conclusion, there exists some constant $c$ independent of $R$ such that

$$
c \epsilon \geq \frac{1}{V_{x_{0}\left(\frac{2 R}{5}\right)}} \int_{E(R, 2 R)} h(x) d x \geq \frac{1}{V_{E}(R, 2 R)} \int_{E(R, 2 R)} h(x) d x .
$$

Since $\epsilon$ is arbitrary,

$$
\lim _{R \rightarrow \infty} \frac{1}{V_{E}(R, 2 R)} \int_{E(R, 2 R)} h(x) d x=0 .
$$

From the above and by the choice of the function $w$, we obtain

$$
\begin{aligned}
\lim _{R \rightarrow \infty} \frac{1}{V_{E}(R, 2 R)} \int_{E(R, 2 R)} f & =\lim _{R \rightarrow \infty} \frac{1}{V_{E}(R, 2 R)} \int_{E(R, 2 R)} w \\
& =\lim _{x \rightarrow \infty, x \in E} w(x) \\
& =\limsup _{x \rightarrow \infty, x \in E} f(x)
\end{aligned}
$$

Thus, the lemma follows.

We recall a localized version of Yau's gradient estimate for harmonic functions which was proved by Cheng and Yau [C-Y] (see also [L-Y]). 
Lemma 2.7. Let $M$ be an n-dimensional complete Riemannian manifold which may have boundary. Suppose $B_{x}(2 R)$ is a geodesic ball centered at $x \in M$ with radius $2 R$. Let us assume that $B_{x}(2 R) \cap \partial M=\emptyset$. If $f$ is a harmonic function defined on $B_{x}(2 R)$, and if the Ricci curvature of $M$ satisfies Ric $_{M} \geq-k$ on $B_{x}(2 R)$ for some constant $k \geq 0$, then there exists a constant $C>0$ depending only on $n$ such that

$$
|\nabla f|^{2}(y) \leq C\left(R^{-2}+k\right) \max _{B_{x}(2 R)} f^{2}
$$

for all $y \in B_{x}(R)$.

\section{Proof of theorem 1.1.}

Now we are ready to prove theorem 1.1. But first we prove a slightly more general result. In the following, we shall let $C(k, n)$ denote a constant depending on $k$ and $n$, which may change from line to line.

Theorem 3.1. Let $M^{n}$ be a complete noncompact manifold and $p \in M$ be a fixed point such that $\operatorname{Ric}_{M}(x) \geq-k(r(x))$ where $r(x)=r(x, p)$. Suppose that $k:[0, \infty) \rightarrow[0, \infty)$ is a nonincreasing continuous function such that $\int_{0}^{\infty} r^{n-1} k(r) d r<\infty$. Suppose also that the double of each nonparabolic end of $M$ satisfies condition (VC) and has finite first Betti number. Then the space of all linear growth harmonic functions has dimension less than or equal to some constant $C(n, k)$ depending on $n$ and function $k$ only.

Proof. If $n=2$, the theorem follows from [L-T5]. Hence we assume that $n \geq 3$. From the assumption on the Ricci curvature and theorem 2.2 , we know that $M$ has finite many ends. Therefore, there exists some number $a>0$ such that the number of ends corresponding to the geodesic ball $B_{p}(a)$ is that of $M$. Let $e_{1}, \ldots, e_{s}$ denote all the parabolic ends and $E_{1}, \ldots, E_{l}$ all the nonparabolic ends of $M$ corresponding to $B_{p}(a)$. Also by theorem 2.2, we have $l+s \leq C(k, n)$. Let $f$ be a linear growth harmonic function on $M$. Since from the assumption it is easy to see that the Ricci curvature satisfies $\operatorname{Ric}(y) \geq-\frac{C}{r^{2}(x)}$ on $B_{x}\left(\frac{r(x)}{2}\right)$ for some nonnegative constant $C$, applying lemma 2.7 to $f$ on $B_{x}\left(\frac{r(x)}{2}\right)$, we conclude that $|\nabla f|$ is a bounded function on $M$. On the other hand, the Bochner formula together with the Ricci curvature assumption implies that

$$
\triangle|\nabla f|^{2} \geq-k|\nabla f|^{2} \text { on } M
$$


Now we claim that there exists a constant $C(k, n)>0$ such that on each parabolic end $e_{i}$,

$$
\sup _{\partial e_{i}}|\nabla f|^{2} \geq C(k, n) \sup _{e_{i}}|\nabla f|^{2} .
$$

In fact, let $\bar{M}$ be the Ricci model of dimension $n$ (see [G-W]) which has Ricci curvature in the radial direction given by $-k(\bar{r}(\bar{p}, \bar{x}))$, where $\bar{p}$ is the pole of $\bar{M}$ and $\bar{r}$ is the distance function of $\bar{M}$. Let us denote $\bar{A}(r)$ to be the area of $\partial B_{\bar{p}}(r)$. Following [L-T1], we define function $g(x)=\phi(r(x))$ on $M$, where function $\phi$ is given by

$$
\phi(r)=\int_{r}^{\infty} \frac{1}{\bar{A}(s)}\left(\int_{s}^{\infty} \bar{A}(t) k(t) d t\right) d s .
$$

Then it was shown in [L-T1] that $g$ is a well defined nonnegative function on $M \backslash\{p\}$ and satisfies

$$
\triangle g \geq k \text { and } \lim _{x \rightarrow \infty} g(x)=0 .
$$

Let $c_{0}$ be such that $\phi(a) \leq c_{0}$. Note that $c_{0}$ depends only on $k$ and $n$. Also we may choose $a$ sufficiently large such that $c_{0}<1$. Since for $f$ a harmonic function of linear growth, it has bounded gradient as shown above. Thus, for any fixed parabolic end $e$, without loss of generality, we may assume that $\sup _{e}|\nabla f|=1$. Then on $e$, we have by using the Bochner formula,

$$
\triangle\left(|\nabla f|^{2}+g\right) \geq-k|\nabla f|^{2}+k \geq 0 .
$$

If $\sup _{e}\left(|\nabla f|^{2}+g\right)$ is only attained at infinity, then there is a positive superharmonic function defined on $e$ which attains its infimum at infinity. That would imply $e$ is nonparabolic (see [L-T1]), which violates our assumption. In particular, by the maximum principle

$$
\begin{aligned}
\sup _{e}\left(|\nabla f|^{2}+g\right) & \leq \sup _{\partial e}\left(|\nabla f|^{2}+g\right) \\
& \leq \sup _{\partial e}|\nabla f|^{2}+c_{0} \\
& =\sup _{\partial e}|\nabla f|^{2}+c_{0} \sup _{e}|\nabla f|^{2} .
\end{aligned}
$$

Therefore, noting that $c_{0}<1$ and $g$ is nonnegative, we conclude

$$
\sup _{e}|\nabla f| \leq C(k, n) \sup _{\partial e}|\nabla f| .
$$


which is what we have claimed.

On each nonparabolic end $E$, the function $|\nabla f|^{2}+g \sup _{M}|\nabla f|^{2}$ is also bounded and subharmonic. Thus we can apply lemma 2.6 to it. Since $\lim _{x \rightarrow \infty} g(x)=0$, we have

$$
\lim _{R \rightarrow \infty} \frac{1}{V_{E}(R, 2 R)} \int_{E(R, 2 R)}|\nabla f|^{2}=\limsup _{x \rightarrow \infty, x \in E}|\nabla f|^{2} .
$$

Let us now define an inner product on the space of all linear growth harmonic functions which vanish at point $p$ by

$$
\begin{aligned}
& \ll \nabla f, \nabla h \gg=\frac{1}{V_{p}(2 a)} \int_{B_{p}(2 a)}<\nabla f, \nabla h>+ \\
& \quad+\sum_{i=1}^{l} \lim _{R \rightarrow \infty} \frac{1}{V_{E_{i}}(R, 2 R)} \int_{E_{i}(R, 2 R)}<\nabla f, \nabla h>.
\end{aligned}
$$

By (2) and the polarization

$$
2<\nabla f, \nabla h>=|\nabla(f+h)|^{2}-|\nabla f|^{2}-|\nabla h|^{2},
$$

we conclude that all the limits exist. Also, it is easy to see that

$$
\ll \nabla f, \nabla f \gg \leq(l+1)\|\nabla f\|_{\infty}^{2}
$$

On the other hand, the Bochner formula implies that

$$
\triangle|\nabla f|^{2} \geq-C(k)|\nabla f|^{2} \text { on } B_{p}(2 a),
$$

where $C(k)$ is a positive constant depending on function $k$. Therefore,

$$
\left(\triangle-\frac{\partial}{\partial t}\right)\left(e^{-C(k) t}|\nabla f|^{2}\right) \geq 0 \text { on } B_{p}(2 a) .
$$

Using the mean-value inequality in [L-T3], we have

$$
\sup _{B_{p}(a)}|\nabla f|^{2} \leq C(k, n) \frac{1}{V_{p}(2 a)} \int_{B_{p}(2 a)}|\nabla f|^{2} .
$$

Notice that $|\nabla f|^{2}+g \sup _{M}|\nabla f|^{2}$ is subharmonic on $M \backslash B_{p}(a)$. By the maximum principle, the maximum of $|\nabla f|^{2}+g \sup _{M}|\nabla f|^{2}$ is either achieved on $B_{p}(a)$ or at the infinity of $M$. In particular, we have

$$
\begin{aligned}
\sup _{M}|\nabla f|^{2} & \leq \sup _{B_{p}(a)}|\nabla f|^{2}+\sup _{M \backslash B_{p}(a)}\left(|\nabla f|^{2}+g \sup _{M}|\nabla f|^{2}\right) \\
& \leq 2 \sup _{B_{p}(a)}|\nabla f|^{2}+c_{0} \sup _{M}|\nabla f|^{2}+\limsup _{x \rightarrow \infty}|\nabla f|^{2} .
\end{aligned}
$$


Therefore,

$$
\sup _{M}|\nabla f|^{2} \leq C(k, n) \sup _{B_{p}(a)}|\nabla f|^{2}+C(k, n) \limsup _{x \rightarrow \infty}|\nabla f|^{2} .
$$

In view of (1), (2) and (3), we conclude that

$$
\ll \nabla f, \nabla f \gg \geq C(k, n)\|\nabla f\|_{\infty}^{2} .
$$

Thus, $\ll \nabla f, \nabla h \gg$ is indeed an inner product. Let $f_{1}, \ldots, f_{d}$ be orthonormal with respect to this inner product. Following [L-T4], we consider the function

$$
F(y)=\sum_{i=1}^{d} f_{i}^{2}(y),
$$

which is invariant under orthogonal change of basis. Let $H$ be the space spanned by $f_{1}, \ldots, f_{d}$. For any point $y_{0} \in M$, we consider the subspace

$$
H_{0}=\left\{f \in H \mid f\left(y_{0}\right)=0\right\} .
$$

$H_{0}$ must be of at most codimension 1 in $H$ since it is the kernel of the linear functional $L$ which is given by $L(f)=f\left(y_{0}\right)$. Hence, by an orthogonal change of basis for $H$, we can write

$$
F(y)=\sum_{i=1}^{d} \phi_{i}^{2}(y),
$$

where $\phi_{i}\left(y_{0}\right)=0$ for all $i \neq 1$. This implies that

$$
|\nabla F|\left(y_{0}\right) \leq 2\left|\phi_{1}\right|\left(y_{0}\right)\left|\nabla \phi_{1}\right|\left(y_{0}\right) \text {. }
$$

However, $\ll \nabla \phi_{1}, \nabla \phi_{1} \gg=1$ as $\phi_{i}$ 's are orthonomal. Therefore, by (4), we conclude that

$$
\left|\phi_{1}\left(y_{0}\right)\right| \leq C(k, n) r\left(y_{0}\right) \text { and }|\nabla F|\left(y_{0}\right) \leq C(k, n) r\left(y_{0}\right),
$$

where $r$ is the Riemannian distance from $p$. For any compact domain $D \subset M$ with Lipschitz continuous boundary, we have

$$
\begin{aligned}
2 \int_{D}\left(\sum_{i=1}^{d}\left|\nabla f_{i}\right|^{2}\right) & =\int_{D} \Delta\left(\sum_{i=1}^{d} f_{i}^{2}\right) \\
& =\int_{\partial D} \frac{\partial}{\partial r}\left(\sum_{i=1}^{d} f_{i}^{2}\right) \\
& \leq \int_{\partial D}\left|\nabla\left(\sum_{i=1}^{d} f_{i}^{2}\right)\right|
\end{aligned}
$$


If we let $D$ be $B_{p}(2 a)$ and use (5), then we obtain

$$
\int_{B_{p}(2 a)}\left(\sum_{i=1}^{d}\left|\nabla f_{i}\right|^{2}\right) \leq C(k, n) A\left(\partial B_{p}(2 a)\right),
$$

where $A\left(\partial B_{p}(2 a)\right)$ is the area of the boundary of the geodesic ball $B_{p}(2 a)$. For each nonparabolic end $E$, by setting $D$ to be $E(R, 2 R)$ in the above, we also have

(7) $\int_{E(R, 2 R)}\left(\sum_{i=1}^{d}\left|\nabla f_{i}\right|^{2}\right) \leq C(k, n)\left(R A(\partial E(R))+R A\left(E(R) \cap \partial B_{p}(2 R)\right)\right)$,

where $A(\partial E(R))$ and $A\left(E(R) \cap \partial B_{p}(2 R)\right)$ denote the area of the boundary of the sets $E(R)$ and $E(R) \cap B_{p}(2 R)$ respectively. But $V_{E}(R, 2 R) \geq V_{x}\left(\frac{R}{5}\right)$ for $x \in \partial E\left(\frac{3 R}{2}\right)$, and by [L-T2],

$$
V_{E}\left(\frac{3 R}{2}\right) \leq C(k, n) V_{x}\left(\frac{R}{5}\right),
$$

where $V_{E}(r)$ denotes the volume of the set $E \cap B_{p}(r)$. Hence,

$$
V_{E}(R, 2 R) \geq C(k, n) V_{E}\left(\frac{3 R}{2}\right) \geq C(k, n) V_{E}(R) .
$$

Consider $r(x)=r(x, p)$, then by $[\mathrm{Y}], \Delta r \leq \frac{C(n)}{r}$ in the distribution sense for $r$ sufficiently large. In particular, if we integrate this inequality over the set $E \cap B_{p}(r)$ and use integration by parts, we obtain

$$
\left.r A\left(E \cap \partial B_{p}(r)\right)\right) \leq r A(\partial E)+C(n) V_{E}(r) .
$$

Similarly,

$$
R A\left(E(R) \cap \partial B_{p}(2 R)\right) \leq R A(\partial E(R))+C(n) V_{E}(R, 2 R) .
$$

But $E$ is a nonparabolic end, $V_{E}(r) \geq C r^{2}$ (see [C-Y]). Thus, (9) can be written into

$$
\left.r A\left(E \cap \partial B_{p}(r)\right)\right) \leq C(k, n) V_{E}(r) .
$$

From (7), noting (8), (10) and (11), we have

$$
\int_{E(R, 2 R)}\left(\sum_{i=1}^{d}\left|\nabla f_{i}\right|^{2}\right) \leq C(k, n) V_{E}(R, 2 R) .
$$


Since (12) is true for each nonparabolic end $E$ and all large $R$, together with (6), we arrived at

$$
d=\sum_{i=1}^{d} \ll \nabla f_{i}, \nabla f_{i} \gg \leq C(k, n) .
$$

The theorem is proved.

Proof of theorem 1.1. It has been shown in [L-T2] that the condition (VC) is always satisfied on each end of a complete manifold with nonnegative Ricci curvature outside a compact set provided the first Betti number of the manifold is also finite. Now theorem 1.1 follows from theorem 3.1.

We mention one more corollary to theorem 3.1, which in particular contains corollary 1.2. Notice that the manifold $M$ below is in particular asymptotically nonnegatively curved in the sense of [K1]. Hence by [K1] $M$ is of finite topological type and by [L-T2] each end of $M$ satisfies condition (VC).

Corollary 3.2. Let $M^{n}$ be a complete noncompact Riemannian manifold and $p \in M$ be a fixed point. Suppose that the sectional curvature $K_{M}(x) \geq$ $-k(r(x))$, where $r(x)=r(x, p)$ and $k:[0, \infty) \rightarrow[0, \infty)$ is a nonincreasing continuous function such that $\int_{0}^{\infty} r^{n-1} k(r) d r<\infty$. Then the space of all linear growth harmonic functions has dimension less than or equal to some constant $C(n, k)$ depending on $n$ and function $k$ only.

\section{References.}

[A] U. Abresch, Lower curvature bounds, Toponogov's theorem and bounded topology, I, Ann. Sci. Ec. Norm. Sup. 19(1985), 651-670.

[B] R. Bartnik, The mass of an asymptotically flat manifold, Comm. Pure and App.Math. 39(1986), 661-693.

[Cai] M. Cai, Ends of Riemannian manifolds with nonnegative Ricci curvature outside a compact set, Bull. AMS 24(1991), 371-377.

[C-Y] S. Y. Cheng and S. T. Yau, Differential equations on Riemannian manifold and their geometric applications, Comm. Pure and App.Math. 28(1975), 333354 .

[D] H. Donnelly, Bounded harmonic functions and positive Ricci curvature, Math. Z. 191(1986), 559-565. 
[G-W] R. E. Green and H. Wu, Function theory on Manifolds which posses a pole, Lecture Notes in Math. 699, Springer-Verlag 1979.

[H] A. Huber, On subharmonic functions and differential geometry in the large, Comment. Math. Helv. 32(1957), 13-72.

[K1] A. Kasue, A compactification of a manifold with asymptotically nonnegative curvature, Ann. Sci. Ecole. Norm. Sup. 21(1988), 593-622.

[K2] A. Kasue, Harmonic functons with growth conditions on a manifold of asymptotically nonnegative curvature, I, Preprint.

[K3] A. Kasue, Harmonic functons with growth conditions on a manifold of asymptotically nonnegative curvature, II, Recent Topics in Differential and Analytic Geometry, Adv. Stud. Pure Math., no. 18, North-Holland, 1989.

[L1] P. Li, The theory of harmonic functions and its relation to geometry, Proc. Symposia Pure Math. 54(1993), Part I 307-315.

[L2] P. Li, Large time behavior of the heat equation on complete manifolds with non-negative Ricci curvature, Ann. Math. 124(1986), 1-21.

[Liu] Z-D. Liu, Ball covering on manifolds with nonnegative Ricci curvature near infinity, preprint.

[L-T1] P. Li and L. Tam, Harmonic functions and the structure of complete manifolds, J. Diff. Geom. 35(1992), 359-383.

[L-T2] P. Li and L. Tam, Green's Functions, Harmonic Functions and Volume Comparison, preprint (1993).

[L-T3] P. Li and L. Tam, The heat equation and harmonic maps of complete manifolds, Invent. Math. 105(1991), 1-46.

[L-T4] P. Li and L. Tam, Linear growth harmonic functions on a complete manifold, J. Diff. Geom. 29(1989), 421-425.

[L-T5] P. Li and L. Tam, Complete surfaces with finite total curvature, J. Diff. Geom. 33(1991), 139-168.

[L-T6] P. Li and L. Tam, Positive harmonic functions on complete manifolds with non-negative curvature outside a compact set, Ann. Math. 125(1987), 171-207.

[L-Y] P. Li and S. T. Yau, On the parabolic kernel of Schrödinger operator; Acta Math. 156(1986), 153-201.

[SC] L. Saloff-Coste, Uniformly elliptic operators on Riemannian manifolds, J. Diff.Geom. 36(1992), 417-450. 
[T] L. F. Tam, Harmonic functions on connected sums of manifolds, Math. Z. 211(1992), 315-322.

[Y] S. T. Yau, Harmonic functions on complete Riemannian manifolds, Comm. Pure Appl. Math. 28(1975), 201-228.

ReCEIVEd February 2ND, 1995.

STANFORD UNIVERSITY

STANFORD, CA 94305

E-MAIL: JWANG@MATH.STANFORD.EDU 